\title{
An Externalities-Based Decentralized Optimal Power Allocation Algorithm for Wireless Networks
}

\author{
Shrutivandana Sharma and Demosthenis Teneketzis, Fellow, IEEE
}

\begin{abstract}
The rapidly growing demand for wireless communication makes efficient power allocation a critical factor in the network's efficient operation. Power allocation in decentralized wireless systems, where the transmission of a user creates interference to other users and directly affects their utilities, has been recently studied by pricing methods. However, pricing methods do not result in efficient/optimal power allocations for such systems for the following reason. Systems where a user's actions directly affect the utilities of other users are known to have externalities. It is well known from Mas-Colell et al. that in systems with externalities, standard efficiency theorems on market equilibrium do not apply and pricing methods do not result in Pareto optimal outcomes. In this paper, we formulate the power allocation problem for a wireless network as an allocation problem with "externalities." We consider a system where each user knows only its own utility and the channel gains from the transmitters of other users to its own receiver. The system has multiple interference temperature constraints to control interference. We present a decentralized algorithm to allocate transmission powers to the users. The algorithm takes into account the externality generated to the other users by the transmission of each user, satisfies the informational constraints of the system, overcomes the inefficiency of pricing mechanisms and guarantees convergence to globally optimal power allocations.
\end{abstract}

Index Terms-Decentralized algorithm, externalities, interference, interference temperature constraint, mechanism design, microeconomics, power allocation, wireless network.

\section{INTRODUCTION}

\section{A. Overview-Literature Survey}

W ITH rapidly growing demand for wireless communication the need for efficient use of spectrum has drawn the attention of researchers, as well as spectrum policy makers. One of the factors that governs the efficiency of spectrum usage is interference control. In order to have interference control in the new spectrum usage standards, it is required that these standards use efficient power allocation mechanisms which optimize the performance of wireless systems keeping the interference level low. The growth in the size of wireless networks also makes it

Manuscript received February 22, 2007; revised October 31, 2007 and February 25, 2009; approved by IEEE/ACM TRANSACTIONS ON NETWORKING Editor N. Shroff. First published August 04, 2009; current version published December 16, 2009. This work was supported in part by NSF Grant CCR-0325571 and NASA Grant NNX-06D47G.

The authors are with the Department of Electrical Engineering and Computer Science, University of Michigan, Ann Arbor, MI 48109 USA (e-mail: svandana@eecs.umich.edu; teneket@eecs.umich.edu).

Color versions of one or more of the figures in this paper are available online at http://ieeexplore.ieee.org.

Digital Object Identifier 10.1109/TNET.2009.2020162 desirable to use decentralized mechanisms for power allocation because centrally controlled mechanisms involve added infrastructure and network vulnerability.

Decentralized mechanisms for power allocation/control in wireless networks have received considerable attention in the literature. These mechanisms can be classified according to their application and the structure of the underlying network Wireless networks can be broadly classified into two types; networks with hierarchical structure and networks without hierarchical structure. In networks with hierarchical structure, users communicate with each other via one or more central entities (called base stations in wireless cellular networks), and these central entities often play an important role in determining the power allocation. The hierarchical structure in cellular networks can further be classified as uplink or downlink based on whether the communication is from the users to a base station or vice versa. In networks without hierarchy, users communicate with one another directly without any central entity. Examples of such networks are ad hoc or mesh networks. Below, we present a brief summary of the existing work on decentralized power allocation for voice and data networks. Within each category, we present existing results for hierarchical and nonhierarchical network structures.

One of the most well-known decentralized algorithms for power control in fixed data rate cellular voice network was proposed by Foschini and Miljanic in [2]. The algorithm proposed in [2] requires only local measurements and achieves the desired minimum signal-to-interference ratio (SIR) for each user with exponentially fast convergence if there exist power levels that meet these requirements for the SIRs. Later in [3], the power control problem similar to that of [2] was formulated as a utility maximization problem by replacing the hard SIR constraints of [2] with sigmoid utilities. With this modification, the algorithm proposed in [3] overcomes the difficulty of divergence which occurs in the algorithm of [2] when there are no feasible power levels that can attain the desired SIRs. The algorithm of [3] also has the flexibility to be tuned for both voice and data services. Lately, in [4] the Foschini-Miljanic algorithm of [2] was generalized for time varying channels and ad hoc networks.

For wireless data networks, results on decentralized mechanisms for uplink power control can be found in [5]-[9]. In [6], the problem of uplink power control in a single cell code division multiple access (CDMA) data network was formulated as a utility maximization problem. An uplink problem similar to that of [6] with SIR based utilities was also investigated in [7]; in this paper, the existence of an equilibrium was shown, and an algorithm for solving the decentralized power control problem was suggested. The problem formulated in [6] was reinvestigated in 
[8] using pricing; it was shown that pricing results in multiple equilibria that are Pareto superior to the equilibria obtained in [6] and [7]. Pricing-based analysis of the uplink power control problem was also done in [9] by introducing user specific parametric utility functions. The authors of [9] proposed two decentralized algorithms, the parallel update and the random update algorithms, that converge to the unique equilibrium of the problem. Work on pricing for downlink CDMA data networks can be found in [10], [11], and [14]. In [10] and [11], optimal resource allocation strategies were determined for a single-class CDMA system for the case when the utility functions of the users are common knowledge (see [12] and [13] for the definition of common knowledge). In [14], the downlink power allocation problem for multiclass CDMA networks was studied by a decentralized mechanism based on dynamic pricing and partial cooperation between mobiles and the base station. This mechanism achieves a partial-cooperative optimal power allocation that was shown to be close to a globally optimal power allocation. In [15], pricing ideas were used for power allocation in wireless CDMA data networks having a mesh structure. The authors studied power allocation under an interference temperature constraint (ITC) and proposed two auction-based power allocation mechanisms. Under certain conditions, the SIR auction of [15] achieves a power allocation arbitrarily close to a Pareto optimal one, and the power auction achieves an allocation arbitrarily close to the socially optimal one. These conditions, however, require in essence that the manager, which governs the power allocation, should know the users' utility functions.

In this paper, we consider a power allocation problem for a wireless network where the users' transmissions create interference to other users and the system has multiple interference temperature constraints to control the interference. We consider a decentralized network where each user knows only its own utility function, the channel gains from all the transmitters to its own receiver, and the upper and lower bounds on the possible transmission powers of all the users. For this decentralized network, the objective is to determine power allocations that satisfy the interference temperature constraints and maximize the sum of the users' utilities. Below, we explain the motivation for considering this problem.

\section{B. Motivation}

A wireless network is said to have externalities when: 1) each user's transmission creates interference to other users; and 2) each user's utility is directly affected by the interference. Power allocation problems in wireless networks where externalities are present were previously considered in [6]-[9], [14], and [15]. In [6]-[9], power allocation problem is formulated as a noncooperative game, and in [14] and [15], power allocation problem is formulated as a social welfare maximization problem. The solution approach in all of the above references is based on different variations of pricing mechanisms. The pricing mechanisms employed in [6]-[8] and [15] do not achieve globally optimal allocations; the pricing mechanism in [9] does not achieve optimal allocations unless the users vary their utilities according to their target signal to interference ratios; and the pricing mechanism proposed in [14] obtains close to globally optimal allocations.
The reason why the pricing mechanism proposed in [14] results in a globally optimal allocation is the following. The authors of [14] introduce a constraint on the total power transmitted by the base station. Due to this constraint, the original problem, where each user's utility depends on everyone's transmission power, reduces to one where each user's utility depends only on the power allocated to it. Thus, the externalities due to interference that are present in the original problem formulated in [14] disappear, and the pricing mechanism proposed in [14] results in efficient allocations. For the cases where the system has either no maximum power constraint or has multiple power constraints, the above-stated reduction is not possible.

In general, in decentralized resource allocation problems with externalities (i.e., in problems where the resources allocated to each user directly affect the utility of every other user), pricing mechanisms fail to obtain globally optimal allocations. This fact is well known in the economics literature (see [1, Ch. 10]) and has also been identified by the authors of [8] in the context of power allocation in wireless networks. The decentralized resource allocation problem for an economy with externalities was studied by Reichelstein in [16]. Under the assumption that the users of the system cooperate and obey the rules of the mechanism, Reichelstein determined a lower bound on the dimensionality of the message space $^{1}$ required by any mechanism so as to achieve globally optimal allocations. For a system with $M$ users, this lower bound is of the order $O\left(M^{2}\right)$. In game-theoretic formulations of problems with externalities, the message space required by any mechanism to obtain globally optimal allocations is of even higher dimension (see the discussion in [17]-[19]). On the other hand, the dimensionality of the message space of pricing mechanisms (including those in the aforementioned communication networks literature) is of the order $O(M)$. Thus, the information exchanged among the users in the mechanisms proposed in the aforementioned literature is not sufficient (rich enough) to lead to globally optimal allocations. The failure of pricing mechanisms to produce globally optimal solutions of power allocation problems where the users' utilities are directly affected by the interference provides the key motivation for the formulation and solution methodology presented in this paper for power allocation problems in wireless networks.

\section{Contributions of the Paper}

The key contributions of this paper are: 1) the formulation of power allocation problem for wireless networks with interference as an allocation problem with externalities; and 2) the discovery of a decentralized power allocation algorithm, based on the externalities formulation, that results in globally optimal power allocations.

Our formulation properly captures and directly addresses the effect of transmission power externalities on the system performance. Our problem formulation and the proposed power allocation algorithm are distinctly different from all previous studies of utility-based power allocation problems because the previous studies employ pricing mechanisms. The algorithm proposed in this paper leads to a globally optimal power allocation under the assumption that the rules of the algorithm are obeyed by the

1i.e., the space of communication language used by the users to communicate with one another. 


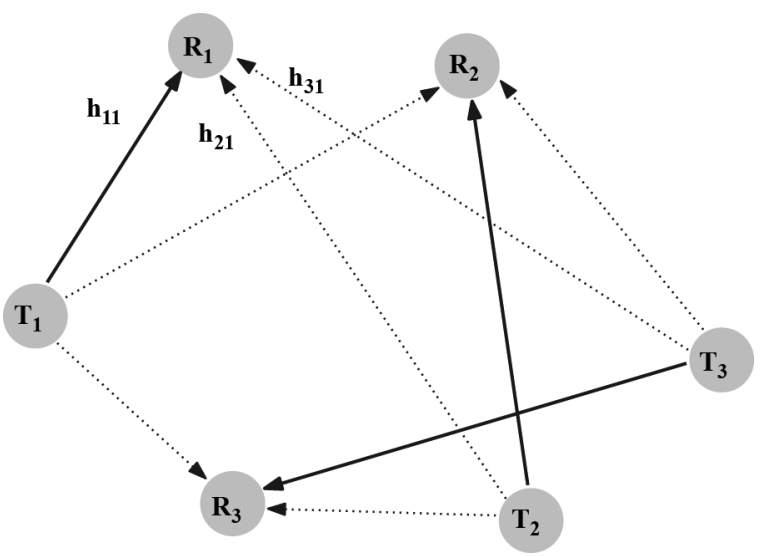

Fig. 1. An example of a wireless mesh network with three users (pairs of nodes); $T_{i}$ and $R_{i}$ denote the transmitter and the receiver of user $i$, respectively, and $h_{i j}$ is the channel gain from $T_{i}$ to $R_{j}$.

users. Thus, our approach to the power allocation problem is non-game-theoretic. The message space of the proposed algorithm has dimension $M^{2}$ and, thus, has the same order as Reichelstein's lower bound $\left[O\left(M^{2}\right)\right]$. This means that the information exchanged among the users in the proposed algorithm is very close to the minimum information exchange required by any mechanism that achieves globally optimal power allocations. The rest of the paper is organized as follows. In Section II, we present the system model and formulate the power allocation problem as an optimization problem. In Section III-A, we formulate the power allocation problem of Section II as an externality problem. In Section III-B, we present a decentralized algorithm for power allocation based on the formulation of Section III-A, and in Appendix A, we prove that the decentralized algorithm results in a globally optimal allocation. In Section IV, we present numerical results for two practical examples. We conclude in Section V.

\section{The Power Allocation Problem}

We consider a wireless network and formulate a power allocation problem for it. We first describe the model and the assumptions we make for its analysis; we also discuss scenarios that motivate the model. We then formulate the power allocation problem as an optimization problem.

\section{A. The Model}

We consider a wireless network consisting of $M$ transmitter-receiver pairs (connected by solid arrows in Fig. 1). We call each transmitter-receiver pair a user ${ }^{2}$, and we denote the set of all users in the network by $\mathcal{M}:=\{1,2, \ldots, M\}$. The users - in other words, the transmitters and the receivers-can be arbitrarily located in the network. This model captures a wide variety of scenarios, e.g., when the transmitters and receivers are located anywhere in the network, the model can represent a wireless ad hoc network or a segment of a wireless

\footnotetext{
${ }^{2}$ In the rest of the paper, any action by a user associated with the transmission of the signal means that it is done by the corresponding transmitter, and any action/computation associated with the reception of a signal means that it is done by the corresponding receiver.
}

mesh network; if the transmitters are colocated, it can represent a cellular downlink network; and if the receivers are colocated, it can represent a cellular uplink network.

User $i, i \in \mathcal{M}$, transmits with power $p_{i}$. We assume the following.

Assumption 1: Every user's transmission creates interference (shown by the dashed arrows in Fig. 1) to all other users in the network; i.e., the graph in Fig. 1 is fully connected.

Assumption 1 implies that the interference to user $i, i \in \mathcal{M}$, depends on the transmission power $p_{j}, j \in \mathcal{M}, j \neq i$, of all other users. To control interference, the system has interference temperature constraints (ITCs) at $K$ different measurement centers ( $\mathrm{MCs}), \mathrm{MC}_{1}, \mathrm{MC}_{2}, \ldots, \mathrm{MC}_{K}$. Interference temperature (IT) is defined in [20] as the net radio frequency (RF) power measured at a receiving antenna per unit bandwidth; ITC is a constraint that puts an upper limit on the IT. To keep the RF noise floor in a wireless network below a safe threshold, it is desirable that the network satisfies an ITC. In our model, we consider multiple ITCs because multiple ITCs can ensure a balanced interference threshold throughout the network. Each ITC is governed by one of the MCs. The MCs can be installed either at the receivers of the users or there can be separate stations acting as MCs. To simplify the notation, we refer to $\mathrm{MC}_{k}$ as user $0_{k}, k \in \mathcal{K}:=\{1,2, \ldots, K\}$, and we denote the set of MCs by $0_{\mathcal{K}}:=\left\{0_{1}, 0_{2}, \ldots, 0_{K}\right\}$. The ITCs are given by

$$
\sum_{i=1}^{M} p_{i} h_{i 0_{k}} \leq P_{k}, \quad k \in \mathcal{K}
$$

where $h_{i 0_{k}}$ is the channel gain from user $i$ 's transmitter to the $k$ th measurement center. We assume the following.

Assumption 2: Each measurement center $\mathrm{MC}_{k}, k \in \mathcal{K}$, can measure the channel gains $h_{i 0_{k}}, i \in \mathcal{M}$; hence, it knows these channel gains. ${ }^{3}$ However, $\mathrm{MC}_{k}$ need not know the channel gains $h_{i 0_{z}}, i \in \mathcal{M}, z \neq k$, to other MCs.

Because of the presence of interference, the quality of service of a user in such a network depends not only on the power received from its own transmitter, but also on the total interference, which depends on the transmission powers of all other users. Hence, to quantify the users' performance, we associate with each user $i, i \in \mathcal{M}$, a utility function $U_{i}\left(p_{1}, p_{2}, \ldots, p_{M}\right)$, which is a function of the transmission power of all the users. We assume the following.

Assumption 3: The transmission power $p_{i}$ of user $i, i \in \mathcal{M}$, lies in $\mathcal{P}_{i}=\left[0, P_{i}^{\max }\right]$, and the set $\mathcal{P}_{i}$ is user $i$ 's private information; i.e., it is known only to user $i$ and nobody else in the system. Furthermore, a set $\mathcal{P}=\left[0, P^{\max }\right] \supset \cup_{i \in \mathcal{M}} \mathcal{P}_{i}$ is common knowledge to all the users (including the MCs).

Unlike active users $i \in \mathcal{M}$, the MCs do not receive any personal benefits from the power allocation; therefore, we assume that the MCs have zero utilities,

\section{Assumption 4:}

$$
U_{0_{k}}(p)=0, \forall k \in \mathcal{K}
$$

${ }^{3}$ We assume that the users in the network are cooperative. Therefore, the trans mitters can periodically transmit pilot signals with known amplitudes; the receivers at the MCs can then measure the received amplitudes from which they can compute the channel gains. 
where

$$
p:=\left(p_{1}, p_{2}, \ldots, p_{M}\right)
$$

For users $i \in \mathcal{M}$, we assume the following.

Assumption 5: $U_{i}(p), i \in \mathcal{M}$, from $R^{M}$ into $R$ is a strictly concave, continuous function of $p$.

In Appendix B, we present an example of a utility function that satisfies Assumption 5.

We also assume the following.

Assumption 6: Each user's utility function is its own private information.

The above assumption captures a variety of scenarios. One such scenario is a multimedia wireless communication network where different users run different applications, each application with a different utility associated with it that is known only to the user that runs the application. Another possible scenario is where the received data is processed/decoded by different users using different technologies that are not public information. In either scenario, we assume that it is not feasible for the users on informational grounds to communicate their exact utility functions to other users in the network. ${ }^{4}$

Because of Assumption 1, every user can hear every other user in the network; therefore, we assume the following.

Assumption 7: The number of participating users (including the MCs) $M+K$ is common knowledge. We also assume that the number of active users $M$ remains constant during a power allocation period.

If the time scale in which a power allocation is determined is sufficiently small, the system can be assumed to be static for an allocation period; therefore, we assume the following.

Assumption 8: The channel gains $h_{i 0_{k}}, i \in \mathcal{M}, k \in \mathcal{K}$, and the utilities of the users remain constant during a power allocation period.

For the network model presented above, we want to study a power allocation problem that we describe in the next section. Before describing the power allocation problem, it is important to note that the wireless network under consideration is a decentralized information network. This can be seen from Assumptions 2, 3, and 6, which describe the informational constraints of the network. In our problem formulation and solution that follows, we assume that the users in this decentralized network cooperate to jointly arrive at an optimal solution. ${ }^{5}$

\section{B. Optimization Problem}

For the wireless network described in the previous section, the objective is to determine the users' transmission powers under the constraints imposed by the model so as to maximize the sum

\footnotetext{
${ }^{4}$ Since each user's utility function is concave, it is generally parameterized by an infinite number of parameters. Furthermore, since certain regularity conditions must be satisfied by the communication rules employed by the users (see [21]), the dimension (see [21]) of the message space (the space used for message exchange) must be infinite. Thus, communication of the users' utility functions is infeasible on informational grounds.

${ }^{5}$ We assume that the users will not cheat with the system by misrepresenting their private information to derive an unfair gain in their favor. The situation where users behave selfishly and have an incentive to cheat will be addressed in a future publication.
}

of users' utilities. We formally write this optimization problem, that we call Problem (P1), as follows:

Problem (P1)

$$
\max _{p} \sum_{i \in \mathcal{M} \cup 0_{\mathcal{K}}} U_{i}(p)
$$

subject to : Assumptions 1-8, and

$p \in S:=\left\{p \mid \sum_{i=1}^{M} p_{i} h_{i 0_{k}} \leq P_{k}, k \in \mathcal{K}, p_{i} \in \mathcal{P}_{i}, \forall i \in \mathcal{M}\right\}$.

Because of Assumptions 2, 3, and 6, Problem (P1) is a decentralized optimization problem; i.e., none of the users in the network has complete information of all the parameters that describe Problem (P1). Our objective is to develop an algorithm that satisfies the above informational constraints of Problem (P1) and obtains optimal solutions of the corresponding centralized problem where one of the users/a center has complete information of all the parameters that describe Problem (P1). The centralized counterpart of Problem (P1) is

$$
\begin{aligned}
& \max _{p} \sum_{i \in \mathcal{M} \cup 0_{\mathcal{K}}} U_{i}(p) \\
& \text { subject to: } \quad p \in S \& \text { Assumptions } 1,4,5,7 \text {, and } 8 .
\end{aligned}
$$

It should be noted that the centralized counterpart of Problem (P1) is a strictly concave optimization problem, and hence, it has a unique optimum solution.

As pointed out in Section I-B, Problem (P1) is a resource allocation problem with externalities. Resource allocation mechanisms for problems with externalities have been investigated by Laffont and St. Pierre in [22] in the context of economic systems. The authors of [22] propose a decentralized mechanism that leads to an optimal allocation for the corresponding centralized economy. This mechanism was based on some earlier work of Lions and Temam [23]. In this paper, we formulate Problem (P1) as an externality problem that is similar to that of [22]; based on this formulation, we develop an algorithm with an appropriate modification of the ideas of [23]. The algorithm we develop obtains an optimal solution of the centralized counterpart of Problem (P1).

In the following section, we formulate Problem (P1) as an externality problem and present a decentralized algorithm (which we call the externality algorithm) that obtains optimal solutions of the centralized counterpart of Problem (P1).

\section{Solution of the Power Allocation Problem}

\section{A. Formulation as an Externality Problem}

From each user's perspective, we divide the allocation variables into two classes: one consisting of allocations for which the user is responsible, and the other consisting of the rest of the allocation variables for which the user is not directly responsible.

Specifically, we associate with user $i$ a variable $p_{i}$, which is the power allocated to/transmitted by user $i$. We also associate 
with user $i$ an external environment $p_{-i}$, which consists of the powers allocated to/transmitted by all other users $j \neq i$. Mathematically, the external environment of user $i$ is defined to be the vector

$$
p_{-i}:=\left(p_{1}, p_{2}, \ldots, p_{i-1}, p_{i+1}, \ldots, p_{M}\right) .
$$

As is clear, $p_{i}$ is under user $i$ 's control, whereas the variables in $p_{-i}$ are controlled by other users. We define a power profile for the network of $M$ users to be the full $M$-dimensional vector

$$
p:=\left(p_{1}, p_{2}, \ldots, p_{M}\right) .
$$

By Assumption 3, $p_{i}$ is constrained to lie in the set $\mathcal{P}_{i}$. In the absence of an exact knowledge of the constraint sets $\mathcal{P}_{j}, j \neq i$, of other users, the set of possible external environments of user $i$ as perceived by $i$ will be

$$
\mathcal{P}^{M-1}=\left\{p_{-i} \mid p_{j} \in \mathcal{P}, j \in \mathcal{M} \backslash\{i\}\right\} .
$$

It should be noted that some of the elements of $\mathcal{P}^{M-1}$ may never actually exist as an external environment of user $i$ because $\mathcal{P}^{M-1}$ also contains elements outside $\prod_{j \in \mathcal{M} \backslash\{i\}} \mathcal{P}_{j}$, which cannot be used by other users. However, since $\mathcal{P}$ is common knowledge, user $i$ knows that its external environment must lie within $\mathcal{P}^{M-1}$. Since $\mathcal{P}^{M-1}$ is a product set of $M-1$ convex and compact sets, it is also convex and compact.

To see the effect of a user's external environment on the choices of $p_{i}$ it can transmit, we first note that the presence of other users does not prohibit user $i$ to use any power in its possible range $\mathcal{P}_{i}$. We call $p_{i}$ to be technically possible for user $i$ if, given the technical constraints of its device and the externalities, it is possible for it to use power $p_{i}$. Thus, for a given external environment $p_{-i}$, any $p_{i} \in \mathcal{P}_{i}$ is technically possible for user $i$. By combining the possible external environments of user $i$ with the corresponding technically possible choices of $p_{i}$ we define the set of power profiles that are technically feasible for user $i, i \in \mathcal{M}$, as

$$
S_{i}:=\left\{p \mid p_{i} \in \mathcal{P}_{i}, \quad p_{-i} \in \mathcal{P}^{M-1}\right\} .
$$

As can be seen, $S_{i}$ is a product set of two convex and compact sets; hence, $S_{i}$ is also convex and compact. The elements in the intersection $\bigcap_{i \in \mathcal{M}} S_{i}$ are the only power profiles that are technically feasible for all the users in the system. Hence, a feasible solution of Problem (P1) must lie in this intersection. However, it should be noted that the technically feasible power profiles in (10) do not ensure that the ITCs in (1) are satisfied.

The responsibility of making sure that the power profiles satisfy the ITCs is given to the MCs (users $0_{k}, k \in \mathcal{K}$ ). Since, by Assumption 2, user $0_{k}$ knows the channel gains $h_{i 0_{k}}, i \in \mathcal{M}$, exactly, it can check whether or not a given power profile satisfies the corresponding ITC in (1). We call a power profile that satisfies the $k$ th ITC as $k$-constraint-feasible. We associate the set $S_{0_{k}}$ of $k$-constraint-feasible power profiles with user $0_{k}, k \in \mathcal{K}$, as follows:

$$
S_{0_{k}}:=\left\{p \mid \sum_{i=1}^{M} p_{i} h_{i 0_{k}} \leq P_{k}, p_{i} \in \mathcal{P} \forall i\right\} .
$$

Since the sets $S_{0_{k}}, k \in \mathcal{K}$, are intersections of half-spaces and are bounded, they are convex and compact. We call a power profile to be constraint-feasible if it is $k$-constraint-feasible for all $k \in \mathcal{K}$. It can be seen from (11) that a constraint-feasible profile is acceptable for the system in terms of satisfying the ITCs, but it may not be technically feasible for all users $i \in \mathcal{M}$ because it does not necessarily satisfy the technical feasibility condition $p_{i} \in \mathcal{P}_{i}, \forall i$. For a power profile to be fully feasible for the system, it must be both constraint-feasible as well as technically feasible for all users $i \in \mathcal{M}$. Mathematically, the set of feasible power profiles can be defined as $S:=\bigcap_{i \in \mathcal{M} \cup 0_{\mathcal{K}}} S_{i}$. It should be noted that $S$ is a nonempty set since $p=0$ is an element of $S_{i} \forall i \in \mathcal{M} \cup 0_{\mathcal{K}}$. Furthermore, $S$ is also convex and compact since it is an intersection of convex and compact sets. Going back to the optimization problem in Section II-B, it can be seen that the set $S$ we have just defined is the same as the set defined in (4) over which the objective function in Problem (P1) has to be optimized. But now, by separating the external environment and private information of each user from those of other users, we have decomposed $S$ into a number of sets, $S_{i}, i \in \mathcal{M} \cup 0_{\mathcal{K}}$, each of which can be associated with an individual user. Furthermore, each set $S_{i}$ is such that user $i$, $i \in \mathcal{M} \cup 0_{\mathcal{K}}$, has complete knowledge of the parameters required to completely describe $S_{i}$. With this decomposition, we are now ready to present an algorithm for solving the power allocation problem presented in Section II-B.

\section{B. Decentralized Optimal Power Allocation Algorithm}

We present a synchronous 6 iterative process, which we call the externality algorithm, that satisfies the informational constraints of Problem (P1) and leads to an optimal solution of the centralized counterpart of Problem (P1).

\section{Externality Algorithm:}

Step 0) Before the start of the iterative process, all users (including $0_{1}, 0_{2}, \ldots, 0_{K}$ ) agree upon a common power profile. This profile can be any arbitrary $p^{(0)} \in\left\{p \mid p_{i} \in \mathcal{P} \forall i\right\}$ that need not necessarily be a constraint-feasible or technically feasible one.

Before the start of the iterative process the users also agree upon $^{7}$ a sequence $\left\{\tau^{(n)}\right\}_{n=1}^{\infty}$ of modification parameters that will be used in the algorithm. The sequence $\tau^{(n)}$ is chosen to satisfy the following three properties:

$$
\begin{aligned}
& 0<\tau^{(n+1)} \leq \tau^{(n)} \leq 1, \forall n \geq 1 \\
& \lim _{n \rightarrow \infty} \tau^{(n)}=0 \\
& \lim _{n \rightarrow \infty} \sigma^{(n)}=\infty
\end{aligned}
$$

where $\sigma^{(n)}:=\sum_{t=1}^{n} \tau^{(t)}$. For instance, $\tau^{(n)}=\frac{1}{n}, n=$ $1,2,3, \ldots$, can be chosen as the sequence. The counting variable $n$ is set to 0 .

\footnotetext{
${ }^{6}$ In each iteration, the message update is done synchronously by all the users.

${ }^{7}$ Since the users have a common objective, they can communicate with one another before the iterative process/algorithm begins and determine $\left\{\tau^{(n)}\right\}_{n=1}^{\infty}$ and $p^{(0)}$ that will be used in the algorithm. Alternatively, $\left\{\tau^{(n)}\right\}_{n=1}^{\infty}$ as well as $p^{(0)}$ can be given to the users by the system designer.
} 
Step 1) At the $n$th iteration, each user $i \in \mathcal{M}$ (respectively, $\left.M C_{k}, k \in \mathcal{K}\right)$ maximizes its $n$th stage payoff on its technically feasible set $S_{i}$ (respectively, the $k$ th-constraint-feasible set $S_{0_{k}}$ ). Specifically, user $i, i \in \mathcal{M}$, solves

$$
\hat{p}_{i}^{(n+1)}=\arg \max _{p \in S_{i}} U_{i}(p)-\frac{1}{\tau^{(n+1)}}\left\|p-p^{(n)}\right\|^{2}
$$

and $M C_{k}, k \in \mathcal{K}$, solves

$$
\hat{p}_{0_{k}}^{(n+1)}=\arg \max _{p \in S_{0_{k}}}-\frac{1}{\tau^{(n+1)}}\left\|p-p^{(n)}\right\|^{2} .
$$

The optimal answers ${ }^{8} \hat{p}_{i}^{(n+1)}, \forall i \in \mathcal{M} \cup 0_{\mathcal{K}}$, are broadcast to all the users in the system.

Step 2) Upon receiving $\hat{p}_{i}^{(n+1)}, i \in \mathcal{M} \cup 0_{\mathcal{K}}$, the users compute the average of all these power profiles

$$
p^{(n+1)}=\frac{1}{M+K} \sum_{i \in \mathcal{M} \cup 0_{\mathcal{K}}} \hat{p}_{i}^{(n+1)} .
$$

Each user $i, i \in \mathcal{M} \cup 0_{\mathcal{K}}$, also computes the following weighted average:

$$
\hat{w}_{i}^{(n+1)}=\frac{1}{\sigma^{(n+1)}} \sum_{t=1}^{n+1} \tau^{(t)} \hat{p}_{i}^{(t)}
$$

where

$$
\sigma^{(n+1)}=\sum_{t=1}^{n+1} \tau^{(t)}=\sigma^{(n)}+\tau^{(n+1)}
$$

The counter $n$ is increased to $n+1$, and the process repeats from Step 1$)$. At the $(n+1)$ th iteration, the average calculated in (17) is used as a reference power profile for maximization in (15) and (16). The new modification parameter, $\tau^{(n+2)}$, for the $(n+1)$ th iteration is selected from the predefined sequence chosen in Step 0).

As stated in Section II-A, the network model considered in this paper can represent both hierarchical as well as nonhierarchical networks. For networks that have hierarchy such as a single cell cellular uplink or downlink network in which all the users and the MCs communicate with one base station that is responsible for power allocation, the externality algorithm is modified as follows. After computing $\hat{p}_{i}^{(n+1)}$ in Step 1), all users and measurement centers send their respective $\hat{p}_{i}^{(n+1)}, i \in \mathcal{M} \cup 0_{\mathcal{K}}$, to the base station. In Step 2) of the algorithm, the base station computes $p^{(n+1)}$ and $\hat{w}_{i}^{(n+1)}$ for some given $i, i \in \mathcal{M} \cup 0_{\mathcal{K}}$; this $i$ remains fixed throughout the algorithm. ${ }^{9}$ Then, the base station announces $p^{(n+1)}$ back to the users; $p^{(n+1)}$ is used by the users as a reference power profile for optimization in Step 1) of the next iteration. With this modification a big part of computations are done at the base station, and each user or measurement center needs to compute only $\hat{p}_{i}^{(n+1)}$ at each iteration of the algorithm.

The externality algorithm has the following feature.

\footnotetext{
${ }^{8}$ Since $S_{i}$ is a compact set and $U_{i}(\cdot)$ is strictly concave, a unique maximum exists for every $i$.

${ }^{9}$ It is sufficient for the base station to compute $\hat{w}_{i}^{(n+1)}$ only for one $i$ since all the sequences $\hat{w}_{i}^{(n+1)}, i \in \mathcal{M} \cup 0_{\mathcal{K}}$, converge to the same limit (see Theorem 1 ), which is the optimal solution of the centralized counterpart of Problem (P1).
}

Theorem 1: The sequences $\left\{\hat{w}_{i}^{(n)}\right\}_{n=1}^{\infty}, i \in \mathcal{M} \cup 0_{\mathcal{K}}$, obtained by the externality algorithm converge to the unique global optimum of the centralized counterpart of Problem (P1).

It should be noted that the dimensionality of the message space required by the externality algorithm for both hierarchical as well as nonhierarchical networks is $M^{2}$, which is same as the order $O\left(M^{2}\right)$ of the lower bound [16] on the dimensionality of the message space required by any mechanism so as to achieve globally optimal power allocations.

The proof of Theorem 1 is given in Appendix A. Below, we present a discussion that explains the intuition behind the externality algorithm.

As stated in Section II-B, our objective in developing the externality algorithm is to come up with a decentralized iterative process that satisfies the constraints (posed by the network model of Section II-A) on the information available to different users and obtains an optimal solution of the centralized counterpart of Problem (P1) in which one of the users (or a center) has complete system information. To accomplish the above objective, the design of the iterative process requires that, at each step, every user must solve an individual optimization problem based only on the information available to it at that step. ${ }^{10}$ Based on the outcome of individual optimization, every user should then send a message that can be used by other users as additional information in the following iterations. Thus, designing an appropriate iterative process breaks down to designing appropriate "individual optimization problems" and "message exchange rules" that lead to the maximization of the system objective function. Below, we discuss how the externality algorithm accomplishes these goals.

Because individual utility functions of the users are conflicting due to interference, letting users maximize their respective utilities will not lead to the maximization of the system objective function (the sum of all users' utilities). Therefore, the objective function for individual optimization problems must be some modification of the users' utility functions that can capture the effect of externalities. The norm square terms in (15) and (16) serve this purpose. The norm square term puts a penalty on user $i$ in proportion to its deviation from the average of everybody's proposal for the optimal power profile; thus, it "pulls" user $i$ 's decision toward the other users' evaluations of $i$; the evaluations of these users incorporate the externalities that $i$ generates to them. Furthermore, since the norm square term contains only those variables that are known ${ }^{11}$ to user $i$, and the set $S_{i}$ over which the optimization is performed is also known to $i$, the optimization problem described by (15) is well defined for each user $i \in \mathcal{M}$. Similarly, since the set $S_{0_{k}}$ is known to $M C_{k}$ (user $0_{k}$ ), the optimization problem described by (16) is well defined for each user $0_{k} \in 0_{\mathcal{K}}$. The results $\hat{p}_{i}^{(n)}, i \in \mathcal{M} \cup 0_{\mathcal{K}}$, of the individual optimization problems described by (15)-(16), which are announced at the end of each iteration, convey how each user valued everybody's transmissions. The average $p^{(n)}$ of everybody's optima conveys the

\footnotetext{
${ }^{10}$ This consists of information available to the user at the beginning of the iterative process and the information gathered by it during the course of the iterative process till that particular iteration.

${ }^{11}$ Note: After the announcement of users' optimal power profile proposals at the $n$th iteration, every user knows $p^{(n)}$ at the $(n+1)$ th iteration.
} 
average system valuation of users' transmissions and, hence, is used as a reference for the next iteration.

A desirable property for any iterative process to be useful is its convergence. This is achieved in the externality algorithm by reducing the value of the modification parameter $\tau^{(n)}$ in each iteration. This increases the penalty of individual user deviation from the average [given by (17)] of the optima of previous iteration. Thus, as the algorithm progresses, the power profile $\hat{p}_{i}^{(n)}$ proposed by user $i, i \in \mathcal{M} \cup 0_{\mathcal{K}}$, gets closer and closer to those proposed by other users and eventually everybody agrees upon a "common" power profile. It should be noted, however, that the objective is not only the convergence of the iterative process, but also the maximization of the system objective function at the point of convergence. As can be noted from (15) and (16), the power profile that the users optima $\hat{p}_{i}^{(n)}, i \in \mathcal{M} \cup 0_{\mathcal{K}}$, converge to need not be a maximizer of the system objective function. The reason is the following. Toward the end of the algorithm, the norm square terms in (15) dominate (since $\tau^{(n)} \rightarrow 0$ ) the utility terms in the individual optimization problems. Thus, for very large $n$, the outcome of each individual optimization problem is very close to the average proposal of the previous iteration. However, these outcomes are not representative of the users' utilities that form the system objective function; thus, even though in the limit the outcomes are equal, the limit point is not optimal. The contribution of the users' utilities is accounted for by the weighted time average $\hat{w}_{i}^{(n)}, i \in \mathcal{M} \cup 0_{\mathcal{K}}$. By taking a weighted average of the individual optima over the entire run of the algorithm, the two contributing components to the system objective are taken into account simultaneously: the individual utilities, which are more prominent in the individual optimization toward the beginning of the iterative process (when $\tau^{(n)}$ is comparatively large), and the externalities, whose effect becomes more prominent in the individual optimization towards the end of the algorithm (when $\tau^{(n)}$ approaches 0 ). The decreasing weights $\tau^{(n)}$ facilitate convergence of each sequence $\hat{w}_{i}^{(n)}$ and provide appropriate balance between the contributions of the above two parameters in the point of convergence, thus making the common point of convergence the global optimum of the system objective function.

In the next section, we present numerical results that demonstrate the performance of the externality algorithm.

\section{NUMERICAL RESULTS}

In this section, we study the impact of various parameters on the performance of the externality algorithm. We study the performance of the algorithm for two types of cellular CDMA systems. We first consider an uplink CDMA system where the base station (BS) employs minimum mean squared error (MMSE) multiuser detection (MUD) to decode the signal of each mobile user. We take the utility of a user to be the negative of the mean squared error (MSE) corresponding to the user less the power loss incurred due to signal transmission. Thus, the utility of user $i, i \in \mathcal{M}$, is given by (see [24])

$$
\begin{aligned}
U_{i}(p) & =-\min _{\boldsymbol{m}_{\boldsymbol{i}^{T} \in R^{1 \times M}}} E\left[\left\|b_{i}-\boldsymbol{m}_{\boldsymbol{i}}^{T} \boldsymbol{y}\right\|^{2}\right]-0.1 p_{i} \\
& =-\left[\left(\boldsymbol{I}+\left(2 / N_{0}\right) \boldsymbol{A R} \boldsymbol{A}\right)^{-1}\right]_{i i}-0.1 p_{i}
\end{aligned}
$$

where $b_{i}$ is the transmitted data symbol of user $i, \boldsymbol{y}$ is the output of the matched filter employed by the BS, $I$ is the identity matrix of size $M \times M, N_{0} / 2$ is the two-sided power spectral density of thermal noise, $\boldsymbol{A}:=\operatorname{diag}\left(A_{1}, A_{2}, \ldots, A_{M}\right)$ is the diagonal matrix consisting of the received amplitudes of users 1 through $M$, and $\boldsymbol{R}$ is the cross-correlation matrix of the users' signature waveforms. The second term in the utility expression represents the input power loss incurred when user $i$ transmits its signal at power $p_{i}$. For small cross correlation of user's waveforms, the utility functions given by (20) are close to concave (see Appendix B), and therefore, the externality algorithm is expected to converge to the optimum centralized power allocation for the above system.

To test the performance of the algorithm, we ran simulations as explained below. For each test case, we distributed the users uniformly in a circular area of radius $5 \mathrm{~m}$ around the BS. We placed three MCs in the system; the first coinciding with the BS and the other two symmetrically placed along a diameter of the circle so that each MC is two-thirds radius away from the BS. We assumed that the maximum transmission power limit of each user is anywhere between $2 \mathrm{~W}$ and $5 \mathrm{~W}$, and the power loss due to propagation is determined by the inverse squared distance between the transmitter and the receiver. We assumed that each user uses a normalized bipolar signature waveform of dimension 6 and arbitrarily picks a waveform from all possible signature waveforms for its data transmission. Thus, the users create interference to each other when their chosen waveforms have the same polarity in one or more signal dimensions. In the simulation results that follow, each point in the plots is obtained by averaging 50 identical independent simulation runs.

In Fig. 2, we compare the performance of the externality algorithm for different modification parameter sequences $\left\{\tau^{(n)}\right\}$. We choose the sequence $\left\{\tau^{(n)}\right\}$ to be of the form $\tau^{(n)}=(1 / n)^{\delta}$ for varying $\delta$. We plot the sum of users' utilities for a three-user network against the number of iterations. As can be seen from Fig. 2, for $\delta=0.001$, the algorithm shows best convergence. For values of $\delta$ smaller than 0.001 , the modification term remains small compared to the users' utilities in the individual optimization; therefore, it takes longer time for the users to agree with other users' proposals and reach the global optimum. On the other hand, for values of $\delta$ greater than 0.001 , the modification term dominates the users' utilities in the individual optimization; therefore, it takes the users longer to move away from the initial point $p^{(0)}$ and reach the global optimum. Thus, for any system, there exists an optimum sequence $\left\{\tau^{(n)}\right\}$ that provides best convergence. The optimum sequence would, however, depend on the numerical value range of the user's utility functions under consideration. Therefore, to obtain best convergence, a sequence suitable for the given system must be used.

In Fig. 3, we compare the performance of the externality algorithm for different SNRs $\left(2 P^{\max } / N_{0}\right)$. For a three-user network, we plot the sum of users' utilities against the number of iterations for various SNRs. As can be seen, the algorithm converges to the centralized optimum for all SNR values. However, since different SNRs result in different numerical values of users' utility functions, the convergence time to the centralized optimum varies in each case and depends on the distance of the initial point from the optimum. 


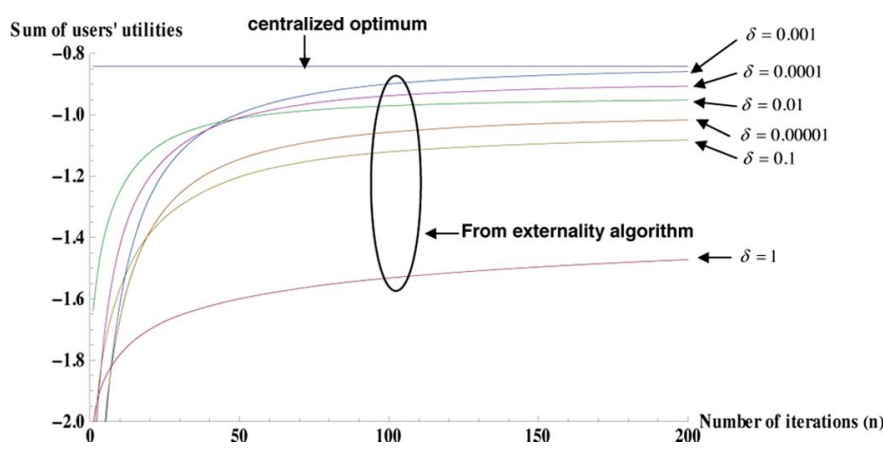

Fig. 2. Sum of users' utilities versus number of iterations for different modification parameter sequences $\left\{\tau^{(n)}\right\}$. Uplink cellular network with three users employing MMSE-MUD; $\tau^{(n)}=(1 / n)^{\delta}, 2 P^{\max } / N_{0}=15 \mathrm{~dB}$.

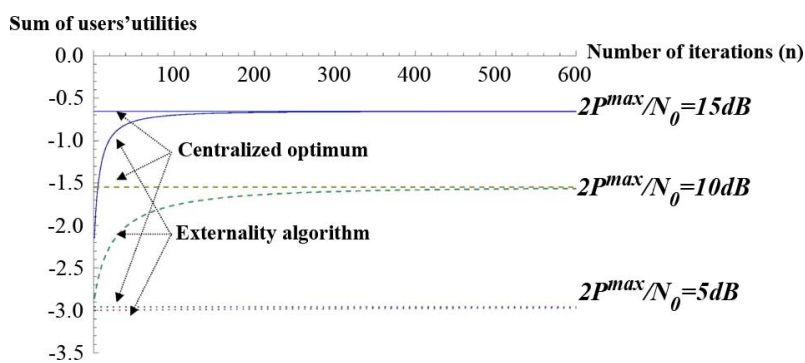

Fig. 3. Sum of users' utilities versus number of iterations for different values of $2 P^{\max } / N_{0}$. Uplink cellular network with three users employing MMSE-MUD; $\tau^{(n)}=(1 / n)^{0.001}$.

For $\tau^{(n)}=(1 / n)^{0.001}$ and $2 P^{\max } / N_{0}=15 \mathrm{~dB}$ that result in best convergence in Figs. 2 and 3, we plot the sequences $\left\{\hat{w}_{i}^{(n)}\right\}$ corresponding to each user $i \in\{1,2,3\}$ and each MC $i \in\left\{0_{1}, 0_{2}, 0_{3}\right\}$ in Fig. 4 . The three components of vectors $\hat{w}_{i}^{(n)}, n=1,2, \ldots$ corresponding to users 1,2 , and 3 are plotted on three separate plots. On the same plots, we also plot the optimum centralized power allocation $\left(p_{1}, p_{2}, p_{3}\right)$. It is clear from the plots that the sequences $\left\{\hat{w}_{i}^{(n)}\right\}$ converge to the centralized optimum that illustrates the statement of Theorem 1.

To compare the performance of the externality algorithm with increasing interference, we plot in Fig. 5 the average utility per user resulting from the externality algorithm against the number of iterations for different number of users. On the same plot, we also show the optimum centralized value of the average utility per user for each case. As can be seen, the algorithm converges to the centralized optimum for three- and four-user case, whereas for six and eight users, it does not converge to the optimum value in given number of iterations. The reason for this is as follows. When the number of users is small compared to the signal dimensions, the cross correlation between the users' waveforms is small; hence, the users' utility functions are close to concave as mentioned in the beginning of Section IV. As the number of users starts overshooting the available signal dimensions, which is six in our simulations, the cross correlation between the users' waveforms increases, and the utility functions are not guaranteed to be close to concave. Therefore, even

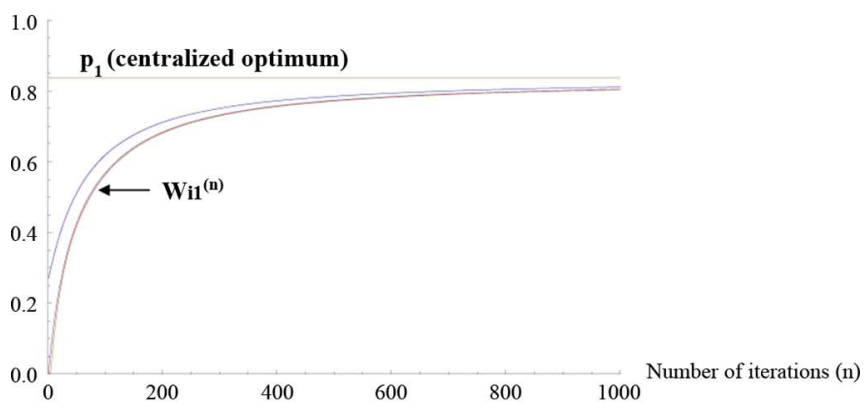

(a)

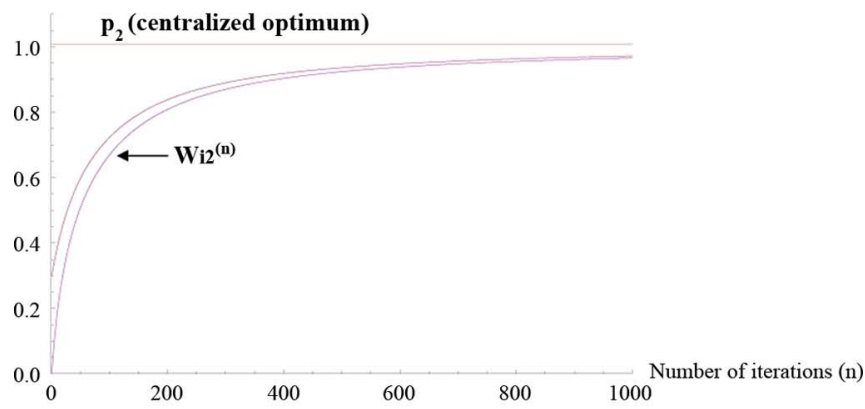

(b)

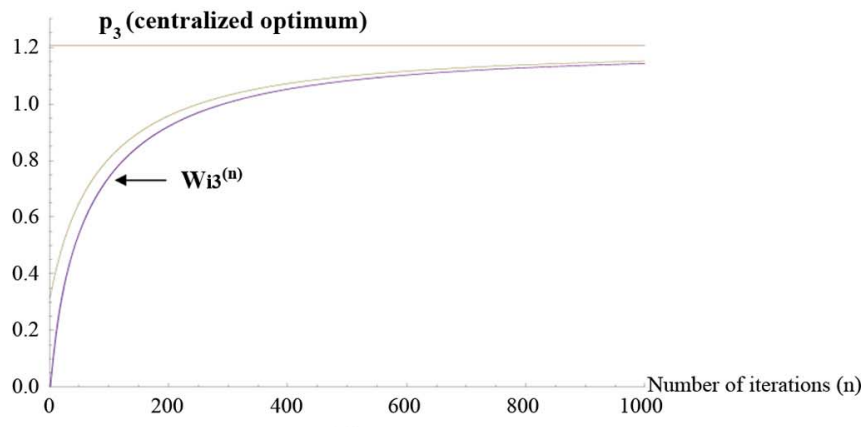

(c)

Fig. 4. Sequence $\left\{\hat{w}_{i}^{(n)}\right\}$ versus number of iterations $n$ for users $i \in\{1,2,3\}$ and MCs $i \in\left\{0_{1}, 0_{2}, 0_{3}\right\}$. Uplink cellular network with three users employing MMSE-MUD; $\tau^{(n)}=(1 / n)^{0.001}, 2 P^{\max } / N_{0}=15 \mathrm{~dB}$.

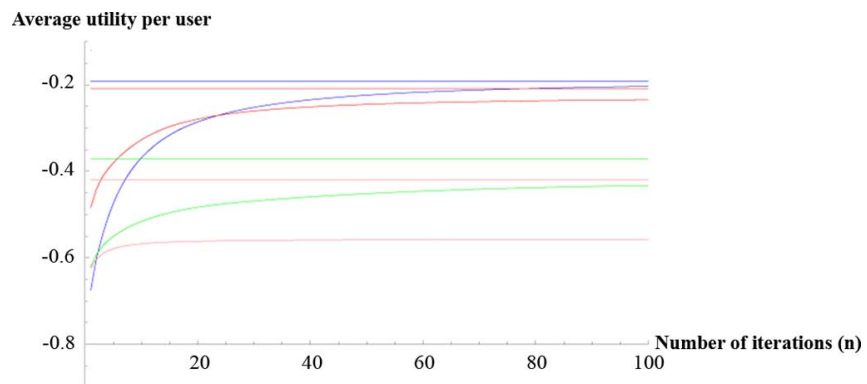

Fig. 5. (color online) Sequence $1 / M \sum_{i \in \mathcal{M} \cup 0 \mathcal{K}} U_{i}\left(w^{(n)}\right)$ versus number of iterations $n$ for 3, 4, 6, and 8 users. Legend: Blue $\rightarrow M=3$, Red $\rightarrow M=$ 4 , Green $\rightarrow \mathrm{M}=6$, Pink $\rightarrow \mathrm{M}=8$. Uplink cellular network employing MMSE-MUD; $\tau^{(n)}=(1 / n)^{0.001}, 2 P^{\max } / N_{0}=15 \mathrm{~dB}$.

though the externality algorithm improves the value of the objective function, it does not converge completely to the global optimum.

Next we consider an uplink CDMA system where the base station (BS) employs simple matched filtering to decode the signal of each mobile user. In this case, we take the utility of 


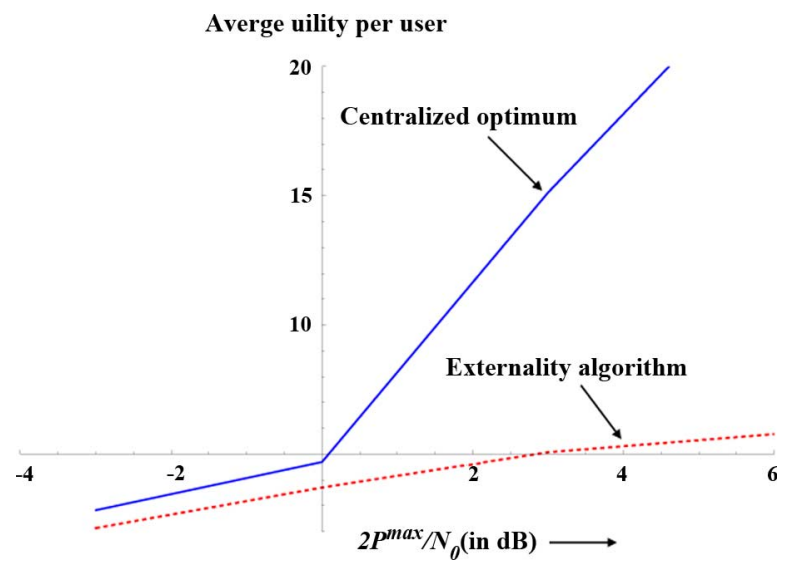

Fig. 6. Average utility per user versus $2 P^{\max } / N_{0}$. Uplink cellular network of 8 users with SINR utility; $\tau^{(n)}=(1 / n)^{0.5}$.

a user to be its signal-to-interference-plus-noise ratio (SINR) at the $\mathrm{BS}$ receiver, i.e.

$$
U_{i}(p)=\frac{p_{i} h_{i 0}}{\frac{N_{0}}{2}+\frac{1}{N} \sum_{j \in \mathcal{M}, j \neq i} p_{j} h_{j 0}}
$$

where $h_{j 0}$ is the channel gain from user $j, j \in \mathcal{M}$, to the BS, and $N$ is the number of dimensions of the users' waveforms. For this system, we set up a simulation scenario in the same way as done for the MMSE-MUD system. We use the same transmission power limits for the users and use the same parameters for the circle radius and propagation loss.

For the SINR utility, the impact of different sequences $\left\{\tau^{(n)}\right\}$ on the convergence of the externality algorithm is similar to that discussed for MMSE-MUD utility. However, the optimum sequence of the form $\tau^{(n)}=(1 / n)^{\delta}$ in this case has $\delta=0.5$. Using this modification sequence, we compare the impact of varying SNR on convergence in Fig. 6. In this figure, we plot two curves representing the average utility per user for an eight-user network. The upper curve shows the optimum centralized value of per-user utility corresponding to each SNR, and the lower curve shows the per-user utility for that SNR at the point of convergence of the externality algorithm. As can be seen, the externality algorithm converges close to the centralized optimum for negative SNR values. For positive SNRs, as the SNR increases, the gap between the centralized optimum and the solution of the externality algorithm increases. The reason for this deviation is that for negative SNRs, the SINR utility is close to concave, but as SNR increases in positive direction, the SINR utility becomes more and more nonconcave; hence, the externality algorithm does not guarantee convergence to the centralized optimum.

The two examples from cellular CDMA communication presented in this section show that the externality algorithm results in optimum centralized power allocation when the networks operate under conditions where users' utilities are close to concave. The modification parameter sequence plays a critical role in determining the convergence speed of the algorithm. Other factors such as SNR and signal dimension impact convergence if they are critical in determining the shape of the utility functions.

\section{CONCLUSION AND Future Work}

Power allocation problems in wireless networks with interference differ from those in wired networks and wireless networks without interference because of the presence of transmission power externalities. Thus, pricing schemes that are useful in developing optimal decentralized power allocation algorithms for networks of latter types do not result in globally optimal allocations for networks with interference. In this paper, we formulated the power allocation problem for a wireless network with interference as an externality problem. We presented a decentralized algorithm that accounts for the transmission power externalities generated by each user to other users. The algorithm results in globally optimal power allocations for concave utility functions and has a message space dimension $M^{2}$ that is of the same order as Reichelstein's lower bound $\left[O\left(M^{2}\right)\right]$.

This work opens up several new directions for further research. Some of the interesting issues that are part of our future investigation are: extension of the externality algorithm to time-varying channels as well as time varying utilities, development of an optimal power allocation algorithm for large networks where every user experiences interference by only a fraction of the users in the network, development of game forms that implement in various types of equilibria the solution of the aforementioned power allocation problems.

\section{APPENDIX A \\ PROOF OF THEOREM 1}

Key Ideas of the Proof of Theorem 1: There are two key steps in the proof of Theorem 1. We first note that each sequence of allocations $\left\{\hat{w}_{i}^{(n)}\right\}_{n=1}^{\infty}, i \in \mathcal{M} \cup 0_{\mathcal{K}}$, generated by the externality algorithm is in a compact set, and therefore, each sequence $\left\{\hat{w}_{i}^{(n)}\right\}_{n=1}^{\infty}$ has a convergent subsequence. In the first step (Claims 1 and 2), we consider such a converging subsequence $\left\{\hat{w}_{i}^{\left(n^{\prime}\right)}\right\}$ of a given user $i$ and the corresponding subsequences $\left\{\hat{w}_{j}^{\left(n^{\prime}\right)}\right\}, j \in\left(\mathcal{M} \cup 0_{\mathcal{K}}\right) \backslash\{i\}$, of all other users. We show that the subsequences $\left\{\hat{w}_{j}^{\left(n^{\prime}\right)}\right\}, j \in\left(\mathcal{M} \cup 0_{\mathcal{K}}\right) \backslash\{i\}$, are also converging subsequences and that all of them converge to the same limit $\hat{w}_{i}^{*}$ as the subsequence $\left\{\hat{w}_{i}^{\left(n^{\prime}\right)}\right\}$. Furthermore, we show that this common limit is a feasible solution of Problem (P1). In the second step (Claim 2 and Claim 3) we show that the aforementioned common limit is an optimal solution of the centralized counterpart of Problem (P1). Since the centralized counterpart of Problem (P1) has a unique optimal solution (as it is a strictly concave optimization problem), and any arbitrarily chosen converging subsequence $\left\{\hat{w}_{i}^{\left(n^{\prime}\right)}\right\}$ of an arbitrarily chosen user $i$ is shown to converge to the optimal solution, it follows that for every $i \in \mathcal{M} \cup 0_{\mathcal{K}}$, all converging subsequences of $\left\{\hat{w}_{i}^{(n)}\right\}_{n=1}^{\infty}$ converge to the optimal solution. This in turn implies that for every $i \in \mathcal{M} \cup 0_{\mathcal{K}}$, the sequences $\left\{\hat{w}_{i}^{(n)}\right\}_{n=1}^{\infty}$ themselves converge to the optimal solution.

Proof of Theorem 1: Let $i \in \mathcal{M} \cup 0_{\mathcal{K}}$ be a given user. Since the set $S_{i}$ is convex and $\hat{p}_{i}^{(t)} \in S_{i}, \forall t$, it follows that the convex combination of $\hat{p}_{i}^{(t)}, \hat{w}_{i}^{(n)}=\frac{1}{\sigma^{(n)}} \sum_{t=1}^{n} \tau^{(t)} \hat{p}_{i}^{(t)} \in S_{i}, \forall n \in$ $\{1,2,3, \ldots\}$. Since $S_{i}$ is compact, there exists a subsequence $\left\{\hat{w}_{i}^{\left(n^{\prime}\right)}\right\}$ of $\left\{\hat{w}_{i}^{(n)}\right\}_{n=1}^{\infty}$ that converges to a limit $\hat{w}_{i}^{*}$ in $S_{i}$. 
Define

$$
w^{(n)}:=\frac{1}{\sigma(n)} \sum_{t=0}^{n-1} \tau^{(t+1)} p^{(t)}
$$

In the following claim, we show that the subsequence $\left\{w^{\left(n^{\prime}\right)}\right\}$ of $\left\{w^{(n)}\right\}_{n=1}^{\infty}$ that is defined by the same set of indices as those of $\left\{\hat{w}_{i}^{\left(n^{\prime}\right)}\right\}$ converges to $\hat{w}_{i}^{*}$. Using this result, we show that the subsequences $\left\{\hat{w}_{j}^{\left(n^{\prime}\right)}\right\}, j \in\left(\mathcal{M} \cup 0_{\mathcal{K}}\right) \backslash\{i\}$, corresponding to users other than $i$ that are specified by the same set of indices as those of $\left\{\hat{w}_{i}^{\left(n^{\prime}\right)}\right\}$, also converge to the same limit-i.e., $\hat{w}_{j}^{\left(n^{\prime}\right)} \rightarrow \hat{w}_{j}^{*}=\hat{w}_{i}^{*}, \forall j \in\left(\mathcal{M} \cup 0_{\mathcal{K}}\right) \backslash\{i\}$.

Claim 1: Let for some $i \in \mathcal{M} \cup 0_{\mathcal{K}}, \lim _{n^{\prime}} \rightarrow \infty \hat{w}_{i}^{\left(n^{\prime}\right)}=$ $\hat{w}_{i}^{*}$. Then

(i) $\lim _{n^{\prime} \rightarrow \infty}\left\|\hat{w}_{i}^{\left(n^{\prime}\right)}-w^{\left(n^{\prime}\right)}\right\|^{2}=0$, i.e., $\lim _{n^{\prime} \rightarrow \infty} w^{\left(n^{\prime}\right)}=\hat{w}_{i}^{*}$

(ii) $\lim _{n^{\prime} \rightarrow \infty}\left\|\hat{w}_{j}^{\left(n^{\prime}\right)}-w^{\left(n^{\prime}\right)}\right\|^{2}=0$, i.e., $\lim _{n^{\prime} \rightarrow \infty} \hat{w}_{j}^{\left(n^{\prime}\right)}=\hat{w}_{i}^{*} \forall j \in\left(\mathcal{M} \cup 0_{\mathcal{K}}\right) \backslash\{i\}$

(iii) The common limit $\hat{w}_{i}^{*}$ of the subsequences $\left\{\hat{w}_{j}^{\left(n^{\prime}\right)}\right\}, j \in$ $\mathcal{M} \cup 0_{\mathcal{K}}$, is a feasible solution of Problem (P1).

Proof: (i) We must show that

$$
\forall \epsilon>0, \exists n_{0}^{\prime}: \forall n^{\prime} \geq n_{0}^{\prime},\left\|\hat{w}_{i}^{\left(n^{\prime}\right)}-w^{\left(n^{\prime}\right)}\right\|^{2} \leq \epsilon .
$$

Since $\|\cdot\|^{2}$ is a convex function, for any $n^{\prime}$

$$
\left\|\hat{w}_{i}^{\left(n^{\prime}\right)}-w^{\left(n^{\prime}\right)}\right\|^{2} \leq \frac{1}{\sigma^{\left(n^{\prime}\right)}} \sum_{t=0}^{n^{\prime}-1} \tau^{(t+1)}\left\|\hat{p}_{i}^{(t+1)}-p^{(t)}\right\|^{2} .
$$

By (12), we have for any $n_{0}<n^{\prime}$

$$
\begin{aligned}
& \frac{1}{\sigma^{\left(n^{\prime}\right)}} \sum_{t=0}^{n^{\prime}-1} \tau^{(t+1)}\left\|\hat{p}_{i}^{(t+1)}-p^{(t)}\right\|^{2} \\
& \leq \frac{1}{\sigma^{\left(n^{\prime}\right)}} \tau^{(1)} \sum_{t=0}^{n_{0}-1}\left\|\hat{p}_{i}^{(t+1)}-p^{(t)}\right\|^{2} \\
& \quad+\tau^{\left(n_{0}\right)} \frac{1}{\sigma^{\left(n^{\prime}\right)}} \sum_{t=n_{0}}^{n^{\prime}-1}\left\|\hat{p}_{i}^{(t+1)}-p^{(t)}\right\|^{2} .
\end{aligned}
$$

In Claim 2, we show that there exists a constant $C_{p} \in(0, \infty)$ independent of $n^{\prime}$ such that

$$
\frac{1}{\sigma^{\left(n^{\prime}\right)}} \sum_{t=n_{0}}^{n^{\prime}-1}\left\|\hat{p}_{i}^{(t+1)}-p^{(t)}\right\|^{2} \leq C_{p} .
$$

Assuming (26) to be true, and given any $\epsilon>0$, we can choose $n_{0}$ (by (13) and [25, Definition 3.1]) such that

$$
\tau^{\left(n_{0}\right)} \leq \frac{\epsilon}{2 C_{p}}
$$

Since $S_{i}, i \in \mathcal{M} \cup 0_{\mathcal{K}}$, is compact and $\hat{p}_{i}^{(t+1)} \in S_{i}$, there exist constants $C_{S_{i}}$ independent of $t$ [25, Theorem 2.41, p. 35] such that

$$
\left\|\hat{p}_{i}^{(t+1)}\right\| \leq C_{S_{i}}, \quad i \in \mathcal{M} \cup 0_{\mathcal{K}} .
$$

Therefore, the sum $A_{0_{i}}$ defined below is finite for any $n_{0}<\infty$ and, in particular, for $n_{0}$ chosen in (27)

$$
A_{0_{i}}:=\sum_{t=0}^{n_{0}-1}\left\|\hat{p}_{i}^{(t+1)}-p^{(t)}\right\|^{2}<\infty .
$$

By (14), $\sigma^{\left(n^{\prime}\right)} \rightarrow \infty$ as $n^{\prime} \rightarrow \infty$; therefore, we can choose an $n_{0_{i}}^{\prime}$ large enough such that

$$
\sigma^{\left(n_{0_{i}}^{\prime}\right)} \geq \frac{2 \tau^{(1)} A_{0_{i}}}{\epsilon}
$$

Then

$$
\begin{aligned}
\forall n^{\prime} \geq n_{0}^{\prime} & :=\max _{i \in \mathcal{M} \cup 0_{\mathcal{K}}} n_{0_{i}}^{\prime}, \\
\sigma^{\left(n^{\prime}\right)} & \geq \sigma^{\left(n_{0}^{\prime}\right)} \geq \sigma^{\left(n_{0_{i}}^{\prime}\right)}, \quad \forall i \in \mathcal{M} \cup 0_{\mathcal{K}} .
\end{aligned}
$$

Substituting (26) and (29) in (25) and using (31) implies that

$$
\begin{aligned}
\left\|\hat{w}_{i}^{\left(n^{\prime}\right)}-w^{\left(n^{\prime}\right)}\right\|^{2} & \leq \frac{1}{\sigma^{\left(n_{0}^{\prime}\right)}} \tau^{(1)} A_{0_{i}}+\tau^{\left(n_{0}\right)} C_{p} \\
& \leq \frac{\epsilon}{2}+\frac{\epsilon}{2}=\epsilon, \quad \forall n^{\prime} \geq n_{0}^{\prime}
\end{aligned}
$$

where the second inequality in (32) follows from (27) and (30). Since $\left\{\hat{w}_{i}^{\left(n^{\prime}\right)}\right\}$ is a converging subsequence with limit $\hat{w}_{i}^{*},(32)$ implies that the subsequence $\left\{w^{\left(n^{\prime}\right)}\right\}$ also converges and has the limit $\hat{w}_{i}^{*}$.

(ii) Replacing $i$ by $j$ in (24)-(32), we obtain for each $j \in$ $\left(\mathcal{M} \cup 0_{\mathcal{K}}\right) \backslash\{i\}$ that

$$
\left\|\hat{w}_{j}^{\left(n^{\prime}\right)}-w^{\left(n^{\prime}\right)}\right\|^{2} \leq \frac{1}{\sigma^{\left(n_{0}^{\prime}\right)}} \tau^{(1)} A_{0_{j}}+\tau^{\left(n_{0}\right)} C_{p} \leq \epsilon .
$$

Since by part (i) $\left\{w^{\left(n^{\prime}\right)}\right\}$ is a converging subsequence with limit $\hat{w}_{i}^{*}$, it follows from (33) that for each $j \in\left(\mathcal{M} \cup 0_{\mathcal{K}}\right) \backslash\{i\}$, $\left\{\hat{w}_{j}^{\left(n^{\prime}\right)}\right\}$ is also a converging subsequence with the limit $\hat{w}_{i}^{*}$.

(iii) Since each set $S_{j}, j \in \mathcal{M} \cup 0_{\mathcal{K}}$, is compact, the limit of each subsequence $\left\{\hat{w}_{j}^{\left(n^{\prime}\right)}\right\}, j \in \mathcal{M} \cup 0_{\mathcal{K}}$, lies in the respective set $S_{j}, j \in \mathcal{M} \cup 0_{\mathcal{K}}$. By parts (i) and (ii), we know that $\forall j \in$ $\mathcal{M} \cup 0_{\mathcal{K}}$, the subsequences $\left\{\hat{w}_{j}^{\left(n^{\prime}\right)}\right\}$ converge to the same limit $\hat{w}_{i}^{*}$. Therefore, by above argument $\hat{w}_{i}^{*} \in S_{j}, \forall j \in \mathcal{M} \cup$ $0_{\mathcal{K}}$. It follows that $\hat{w}_{i}^{*} \in S=\bigcap_{j \in \mathcal{M} \cup 0_{\mathcal{K}}} S_{j}$, and hence, $\hat{w}_{i}^{*}$ is a feasible solution of Problem (P1). To complete the proof of Claim 1, we need to prove (26). This is done in Claim 2.

Claim 2: There exists a constant $0<C_{p}<\infty$ such that

$$
\frac{1}{\sigma^{(n)}} \sum_{t=0}^{n-1}\left\|\hat{p}_{i}^{(t+1)}-p^{(t)}\right\|^{2} \leq C_{p}, \quad \forall n .
$$

Proof: Since $\hat{p}_{i}^{(t+1)}$ is the optimal solution of Step 1) of the algorithm, it follows from [26, Theorem 1.6] that ${ }^{12}$

$$
\begin{aligned}
& \tau^{(t+1)} U_{i}\left(\hat{p}_{i}^{(t+1)}\right)-\left\|\hat{p}_{i}^{(t+1)}-p\right\|^{2} \\
& +\left\|p^{(t)}-p\right\|^{2}-\left\|\hat{p}_{i}^{(t+1)}-p^{(t)}\right\|^{2} \\
& \quad \geq \tau^{(t+1)} U_{i}(p), \forall p \in S_{i}, i \in \mathcal{M} \cup 0_{\mathcal{K}} .
\end{aligned}
$$

${ }^{12}$ by taking $\|\cdot\|^{2}$ as function $J_{1}(\cdot)$ and $U_{i}(\cdot)$ as function $J_{2}(\cdot)$ in Theorem 1.6 of [26] 
Adding (34) over all $i$ implies

$$
\begin{gathered}
\tau^{(t+1)} \sum_{i \in \mathcal{M} \cup 0_{\mathcal{K}}} U_{i}\left(\hat{p}_{i}^{(t+1)}\right)-\sum_{i \in \mathcal{M} \cup 0_{\mathcal{K}}}\left\|\hat{p}_{i}^{(t+1)}-p\right\|^{2} \\
+(M+K)\left\|p^{(t)}-p\right\|^{2}-\sum_{i \in \mathcal{M} \cup 0_{\mathcal{K}}}\left\|\hat{p}_{i}^{(t+1)}-p^{(t)}\right\|^{2} \\
\geq \tau^{(t+1)} \sum_{i \in \mathcal{M} \cup 0_{\mathcal{K}}} U_{i}(p), \forall p \in S:=\bigcap_{i \in \mathcal{M} \cup 0_{\mathcal{K}}} S_{i}
\end{gathered}
$$

By convexity of $\|\cdot\|^{2}$

$$
\left\|p^{(t+1)}-p\right\|^{2} \leq \frac{1}{M+K} \sum_{i \in \mathcal{M} \cup 0_{\mathcal{K}}}\left\|\hat{p}_{i}^{(t+1)}-p\right\|^{2} .
$$

Replacing the second term in (35) using (36), adding (35) over $t=0,1, \ldots, n-1$, and dividing by $M+K$, we get, $\forall p \in S$

$$
\begin{aligned}
& \frac{1}{M+K} \sum_{t=0}^{n-1} \tau^{(t+1)} \sum_{i \in \mathcal{M} \cup 0_{\mathcal{K}}} U_{i}\left(\hat{p}_{i}^{(t+1)}\right)-\left\|p^{(n)}-p\right\|^{2} \\
& -\frac{1}{M+K} \sum_{i \in \mathcal{M} \cup 0_{\mathcal{K}}} \sum_{t=0}^{n-1}\left\|\hat{p}_{i}^{(t+1)}-p^{(t)}\right\|^{2} \\
& \geq \frac{\sigma^{(n)}}{M+K} \sum_{i \in \mathcal{M} \cup 0_{\mathcal{K}}} U_{i}(p)-\left\|p^{(0)}-p\right\|^{2} .
\end{aligned}
$$

By concavity of $U_{i}(p)$ in $p$

$$
\begin{aligned}
& \frac{1}{M+K} \sum_{i \in \mathcal{M} \cup 0_{\mathcal{K}}} \sum_{t=0}^{n-1} \tau^{(t+1)} U_{i}\left(\hat{p}_{i}^{(t+1)}\right) \\
& \leq \frac{\sigma^{(n)}}{M+K} \sum_{i \in \mathcal{M} \cup 0_{\mathcal{K}}} U_{i}\left(\hat{w}_{i}^{(n)}\right)
\end{aligned}
$$

Substituting (38) in (37) and multiplying by $(M+K) / \sigma^{(n)}$, we obtain

$$
\begin{aligned}
& \sum_{i \in \mathcal{M} \cup 0_{\mathcal{K}}} U_{i}\left(\hat{w}_{i}^{(n)}\right)-\frac{M+K}{\sigma^{(n)}}\left\|p^{(n)}-p\right\|^{2} \\
& -\sum_{i \in \mathcal{M} \cup 0_{\mathcal{K}}} \frac{1}{\sigma^{(n)}} \sum_{t=0}^{n-1}\left\|\hat{p}_{i}^{(t+1)}-p^{(t)}\right\|^{2} \\
& \quad \geq \sum_{i \in \mathcal{M} \cup 0_{\mathcal{K}}} U_{i}(p)-\frac{M+K}{\sigma^{(n)}}\left\|p^{(0)}-p\right\|^{2}, \forall p \in S .
\end{aligned}
$$

Since $S_{i}, i \in \mathcal{M} \cup 0_{\mathcal{K}}$, and $S$ are compact, the numerators of the second terms on both the left-hand side (LHS) and the righthand side (RHS) of (39) are bounded. From (14), $\sigma^{(n)} \rightarrow \infty$ as $n \rightarrow \infty$. Therefore

$$
\begin{aligned}
& \lim _{n \rightarrow \infty} \frac{1}{\sigma^{(n)}}\left\|p^{(n)}-p\right\|^{2}=0 \\
& \lim _{n \rightarrow \infty} \frac{1}{\sigma^{(n)}}\left\|p^{(0)}-p\right\|^{2}=0 .
\end{aligned}
$$

Furthermore, since $S_{i}, i \in \mathcal{M} \cup 0_{\mathcal{K}}$, and $S$ are compact, $\hat{w}_{i}^{(n)} \in$ $S_{i}, p \in S$, and $U_{i}(\cdot), i \in \mathcal{M} \cup 0_{\mathcal{K}}$, are continuous functions on
$R^{\mathcal{M}}$, there exist constants $0<C_{U S_{i}}<\infty, i \in \mathcal{M} \cup 0_{\mathcal{K}}$, independent of $n$ such that

$$
\left|U_{i}(p)\right| \leq C_{U S_{i}},\left|U_{i}\left(\hat{w}_{i}^{(n)}\right)\right| \leq C_{U S_{i}}, i \in \mathcal{M} \cup 0_{\mathcal{K}} .
$$

Then, (39) together with (40) -(41) implies that for an appropriate constant $0<C_{p}<\infty$

$$
\frac{1}{\sigma^{(n)}} \sum_{t=0}^{n-1}\left\|\hat{p}_{i}^{(t+1)}-p^{(t)}\right\|^{2} \leq C_{p}, \quad \forall n .
$$

This completes the proof ${ }^{13}$ of Claim 2 and, therefore, the proof of Claim 1.

The arguments used in the proof of Claim 2, specifically those leading to inequality (39), together with Claim 1 allow us to prove that the limit $\hat{w}_{i}^{*}$ is an optimal power allocation.

Claim 3: The limit point $\hat{w}_{i}^{*}$ of the subsequences $\left\{\hat{w}_{j}^{\left(n^{\prime}\right)}\right\}, j \in \mathcal{M} \cup 0_{\mathcal{K}}$, is an optimal solution of the centralized counterpart of Problem (P1).

Proof: From Claim 1, we have that the subsequence $\hat{w}_{j}^{\left(n^{\prime}\right)} \rightarrow \hat{w}_{i}^{*}, \quad \forall j \in \mathcal{M} \cup 0_{\mathcal{K}}$. Therefore, as $n^{\prime} \rightarrow \infty$, the first term on the LHS of (39) converges ${ }^{14}$ to the value of the objective function [in (5)] at $\hat{w}_{i}^{*}$; this can be compared with the value of the objective function at any point $p \in S$ if the limits of the other three terms in (39) are known. From (40), the second terms on both the LHS and the RHS of (39) converge to 0. Since $\|\cdot\|^{2}$ is convex, $\forall i \in \mathcal{M} \cup 0_{\mathcal{K}}$

$$
\begin{aligned}
\left\|\hat{w}_{i}^{\left(n^{\prime}\right)}-w^{\left(n^{\prime}\right)}\right\|^{2} & \leq \frac{1}{\sigma^{\left(n^{\prime}\right)}} \sum_{t=0}^{n^{\prime}-1} \tau^{(t+1)}\left\|\hat{p}_{i}^{(t+1)}-p^{(t)}\right\|^{2} \\
& \leq \frac{1}{\sigma^{\left(n^{\prime}\right)}} \sum_{t=0}^{n^{\prime}-1}\left\|\hat{p}_{i}^{(t+1)}-p^{(t)}\right\|^{2} \\
\because \tau^{(t+1)} & \leq 1, \forall t \geq 0 .
\end{aligned}
$$

Substituting (43) in (39) implies that $\forall p \in S$

$$
\begin{aligned}
& \sum_{i \in \mathcal{M} \cup 0_{\mathcal{K}}} U_{i}\left(\hat{w}_{i}^{\left(n^{\prime}\right)}\right)-\frac{M+K}{\sigma^{\left(n^{\prime}\right)}}\left\|p^{\left(n^{\prime}\right)}-p\right\|^{2} \\
& -\sum_{i \in \mathcal{M} \cup 0_{\mathcal{K}}}\left\|\hat{w}_{i}^{\left(n^{\prime}\right)}-w^{\left(n^{\prime}\right)}\right\|^{2} \\
& \geq \sum_{i \in \mathcal{M} \cup 0_{\mathcal{K}}} U_{i}(p)-\frac{M+K}{\sigma^{\left(n^{\prime}\right)}}\left\|p^{(0)}-p\right\|^{2} .
\end{aligned}
$$

Taking the limit $n^{\prime} \rightarrow \infty$ in (44) and using (32), (33), and (40), we obtain

$$
\sum_{i \in \mathcal{M} \cup 0_{\mathcal{K}}} U_{i}\left(\hat{w}_{i}^{*}\right) \geq \sum_{i \in \mathcal{M} \cup 0_{\mathcal{K}}} U_{i}(p), \forall p \in S .
$$

\footnotetext{
${ }^{13}$ It should be noted that the result of Claim 1 has not been used in the proof of Claim 2. The two claims are presented in the given order only to facilitate the flow of the proof of Theorem 1.

${ }^{14} \mathrm{We}$ only consider the subsequence $\left\{n^{\prime}\right\}$ of $\{n\}$ here, for which $\left\{\hat{w}_{i}^{\left(n^{\prime}\right)}\right\}$ converges.
} 
Claim 4: The sequences $\left\{\hat{w}_{i}^{(n)}\right\}_{n=1}^{\infty}, i \in \mathcal{M} \cup 0_{\mathcal{K}}$, generated by the externality algorithm converge to the optimal solution of the centralized counterpart of Problem (P1).

Proof: In Claims 1-3, we have shown that if we consider any arbitrary converging subsequence $\left\{\hat{w}_{i}^{\left(n^{\prime}\right)}\right\}$ of an arbitrary user $i$, this subsequence converges to an optimal solution $\hat{w}_{i}^{*}$ of the centralized counterpart of Problem (P1). Since the centralized counterpart of Problem (P1) is a strictly concave maximization problem, it has a unique optimal solution $w^{*}$ that must be equal to $\hat{w}_{i}^{*}$. Since the user and the corresponding subsequence are arbitrarily chosen in Claims 1-3, the results of Claims 1-3 hold for all the users and all converging subsequences of each user. This means that for every $i \in \mathcal{M} \cup 0_{\mathcal{K}}$, all converging subsequences of $\left\{\hat{w}_{i}^{(n)}\right\}_{n=1}^{\infty}$ must converge to the unique optimal solution $w^{*}$. Since each sequence $\left\{\hat{w}_{i}^{(n)}\right\}_{n=1}^{\infty}, i \in \mathcal{M} \cup 0_{\mathcal{K}}$, lies in a compact set $S_{i} \subset R^{M}$, and since for each $i \in \mathcal{M} \cup 0_{\mathcal{K}}$, all converging subsequences of $\left\{\hat{w}_{i}^{(n)}\right\}_{n=1}^{\infty}$ converge to the same limit $w^{*}$ (in other words, each sequence $\left\{\hat{w}_{i}^{(n)}\right\}_{n=1}^{\infty}, i \in \mathcal{M} \cup$ $0_{\mathcal{K}}$, has exactly one point of accumulation ${ }^{15}$ ), by [27, Corollary, p. 53], each sequence $\left\{\hat{w}_{i}^{(n)}\right\}_{n=1}^{\infty}$ for $i \in \mathcal{M} \cup 0_{\mathcal{K}}$, itself converges to the optimal solution $w^{*}$.

This completes the proof of Claim 4 and establishes the assertion of Theorem 1.

\section{APPENDIX B}

\section{CONCAVITY OF THE MMSE-MUD UTILITY FUNCTION}

One crucial assumption in Section II-A that is required to prove the convergence of the externality algorithm is that the users' utilities are concave functions of power profiles. In this section, we show the conditions under which the MMSE-MUD utility function studied in Section IV is close to concave.

Suppose there are $M$ users in a network, and all the users use MMSE-MUD receivers to decode the received data. The minimum mean square error at the output of user $i$ 's $(i \in \mathcal{M})$ receiver is then given by (see $[24, \mathrm{Ch} .6]$ )

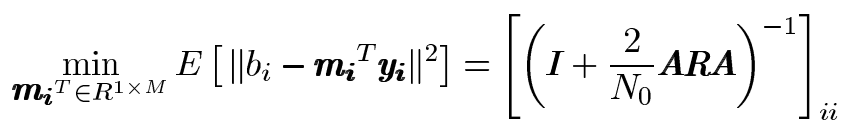

where $b_{i}$ is the transmitted data symbol of user $i, \boldsymbol{y}_{\boldsymbol{i}}$ is the output of user $i$ 's matched filter corresponding to its input received data, $\boldsymbol{I}$ is the identity matrix of size $M \times M, N_{0} / 2$ is the two sided power spectral density of the thermal noise, $\boldsymbol{A}:=$ $\operatorname{diag}\left(A_{1}, A_{2}, \ldots, A_{M}\right)$ is the diagonal matrix consisting of the received amplitudes of users 1 through $M$, and $\boldsymbol{R}$ is the crosscorrelation matrix of the users' signature waveforms. For simplicity of analysis and for analytical tractability, we consider the case of two users below.

For the two-user $(M=2)$ case, the expression for the MMSE in (46) becomes

$$
\begin{aligned}
& \operatorname{MMSE}_{i}=\frac{\frac{N_{0}}{2}}{\frac{N_{0}}{2}+p_{i} h_{i i}\left(1-\frac{\rho^{2}\left(p_{j} h_{j i}\right)}{\left(N_{0} / 2+p_{j} h_{j i}\right)}\right)}, \\
& i, j \in\{1,2\}, j \neq i
\end{aligned}
$$

${ }^{15}$ See [27, Lemma, p. 52].

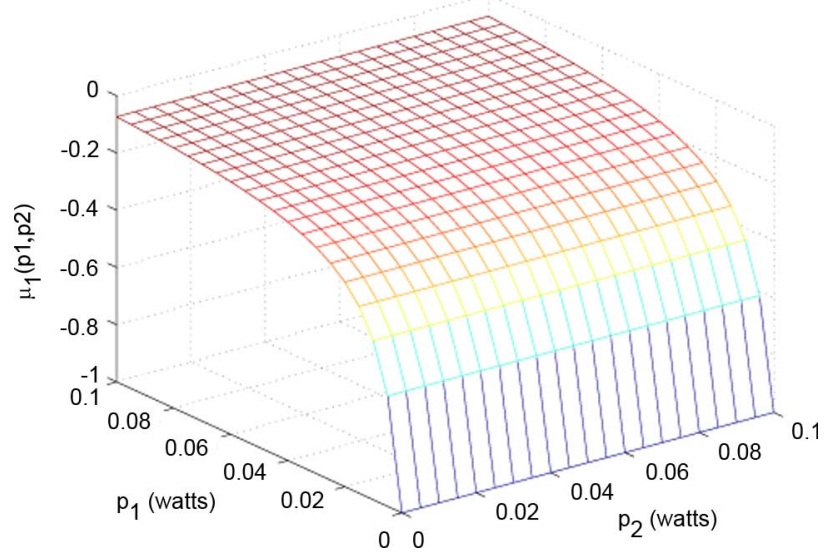

Fig. 7. $U_{1}\left(p_{1}, p_{2}\right)$ versus $\left(p_{1}, p_{2}\right)$ for $N_{0} / 2=10^{-1.2}, h_{11}=0.5, h_{21}=$ 0.6 , and $\rho=0.01$.

where $p_{i} h_{i i}=A_{i}^{2}$ and $p_{j} h_{j i}=A_{j}^{2}, i, j \in\{1,2\}, j \neq i$; $h_{i i}$ and $h_{j i}$ are the channel gains from transmitters $T_{i}$ and $T_{j}$, respectively, to the receiver $R_{i}$; and $\rho$ is the cross correlation between the signature waveforms of users 1 and 2 .

We take the users' utility functions to be

$$
U_{i}(p)=-\operatorname{MMSE}_{i}(p), \quad i \in\{1,2\} .
$$

Below, we investigate the properties of function $U_{i}$ defined in (48). From (47) and (48), we see that for a given PSD $N_{0} / 2$ of the thermal noise, the channel gains $h_{11}, h_{21}$, the cross correlation $\rho$, and the transmission power $p_{2}$ of user 2 , the function $U_{1}$ is of the form $\frac{-1}{c_{1}+c_{2} p_{1}}$ for some constants $c_{1}$ and $c_{2}$. Thus, $U_{1}$ is concave in $p_{1}$. On the other hand, for a given $p_{1}$, if $\rho$ is very small, which is usually the case in practical wireless systems, the coefficient $\rho^{2}$ in the denominator of (47) makes the variation of $U_{1}$ with $p_{2}$ very small. Thus, $p_{1}$ dominantly determines the curvature of function $U_{1}$. To illustrate this, we plot $U_{1}(p)$ versus $\left(p_{1}, p_{2}\right)$ in Fig. 7. It can clearly be seen from Fig. 7 that $U_{1}$ is a concave function of $p_{1}$ and varies very little with $p_{2}$. Therefore, it is close to concave in $p=\left(p_{1}, p_{2}\right)$.

To check the utility of user 2 , we use similar arguments as above by interchanging the indices 1 and 2 , and we get that $U_{2}$ is also close to concave in $p$.

For larger networks with $M>2$, it is difficult to give a general expression for $U_{i}$ similar to (47). However, when the cross correlation among the users' waveforms is small, the curvature of function $U_{i}$ is dominantly determined by $p_{i}$. Similar to the case for $M=2$, the function $U_{i}$ is concave in $p_{i}$ and varies very little with other components of $p$, thus suggesting that it is close to concave in $p$.

\section{ACKNOWLEDGMENT}

The authors are grateful to A. Anastasopoulos for stimulating discussions and to the anonymous reviewers whose comments helped to improve the presentation of the paper.

\section{REFERENCES}

[1] A. Mas-Colell, M. D. Whinston, and J. R. Green, Microeconomic Theory. Oxford, U.K.: Oxford Univ. Press, 2002. 
[2] G. J. Foschini and Z. Miljanic, "A simple distributed autonomous power control algorithm and its convergence," IEEE Trans. Veh. Technol., vol. 42, no. 4, pp. 641-646, Nov. 1993.

[3] M. Xiao, N. B. Shroff, and E. Chong, "Utility-based power control in cellular wireless systems," in Proc. IEEE INFOCOM, Anchorage, AK, 2001, pp. 412-421.

[4] T. Holliday, N. Bambos, P. Glynn, and A. Goldsmith, "Distributed power control for time varying wireless networks: Optimality and convergence," in Proc. 41st Annu. Allerton Conf. Commun., Control, and Comput., Monticello, IL, Oct. 2003, pp. 1024-1033.

[5] C. Saraydar, N. B. Mandayam, and D. J. Goodman, "Pricing and power control in a multicell wireless data network," IEEE J. Sel. Areas Commun., vol. 19, no. 10, pp. 1883-1892, Oct. 2001.

[6] D. G. D. Famolari, N. B. Mandayam, and V. Shah, A New Framework for Power Control in Wireless Data Networks: Games, Utility and Pricing. Boston, MA: Kluwer Academic, 1999.

[7] H. Ji and C. Huang, "Non-cooperative uplink power control in cellular radio systems," Wireless Netw., vol. 4, no. 3, pp. 233-240, Apr. 1998.

[8] C. Saraydar, N. B. Mandayam, and D. J. Goodman, "Efficient power control via pricing in wireless data networks," IEEE Trans. Commun., vol. 50, no. 2, pp. 291-303, Feb. 2002.

[9] T. Alpcan, T. Basar, R. Srikant, and E. Altman, "CDMA uplink power control as a non-cooperative game," Wireless Netw., vol. 8, pp. 659-670, 2002.

[10] P. Liu, M. Honig, and S. Jordan, "Forward-link CDMA resource allocation based on pricing," in Proc. IEEE Wireless Commun. Netw. Conf., Chicago, IL, Sep. 2000, pp. 1410-1414.

[11] C. Zhou, M. Honig, and S. Jordan, "Two-cell power allocation for wireless data based on pricing," in Proc. Allerton Conf. Commun., Control Comput., Monticello, IL, Sep. 2001, vol. 13, pp. 1176-1188.

[12] R. Aumann, "Agreeing to disagree," Ann. Statist., vol. 4, no. 6, pp. 1236-1239, 1976.

[13] R. Washburn and D. Teneketzis, "Asymptotic agreement among communicating decision makers," Stochastics, vol. 13, pp. 103-129, 1984.

[14] J. W. Lee, R. R. Mazumdar, and N. B. Shroff, "Downlink power allocation for multi-class CDMA wireless networks," in Proc. IEEE INFOCOM, 2002, pp. 1480-1489.

[15] J. Huang, R. A. Berry, and M. L. Honig, Auction-based Spectrum Sharing, unpublished.

[16] S. Reichelstein, "Information and Incentives in Economic Organizations," Ph.D. dissertation, Northwestern Univ., Evanston, IL, 1984.

[17] T. Palfrey and S. Srivastava, "Bayesian implementation," in Fundamentals of Pure and Applied Economics 53. New York: Harwood.

[18] E. Maskin, "The theory of implementation in nash equilibrium: A survey," in Social Goals and Social Organization, L. Hurwicz, D. Schmeidler, and H. Sonnenschein, Eds. Cambridge, U.K.: Cambridge Univ. Press.

[19] T. Stoenescu and D. Teneketzis, "Minimal message space Nash implementation of unicast resource allcoation problems in networks", to be published.
[20] "Spectrum policy task force report," Fed. Commun. Commission, 2002.

[21] L. Hurwicz, "On informational decentralization and efficiency in resource allocation mechanisms," Studies in Math. Econom., MAA studies Math., vol. 25, pp. 238-350, 1986.

[22] J. J. Laffont and P. S. Pierre, "Planning with externalities," Int. Econ. Rev., vol. 20, no. 3, pp. 617-634, Oct. 1979.

[23] J. L. Lions and R. Temam, "Eclatement et decentralisation en calcul des variations," in Compte rendu de l'Academie des Sciences, Paris, France, 1971, vol. 263, pp. 563-565.

[24] S. Verdu, Multiuser Detection. Cambridge, U.K.: Cambridge Univ. Press, 2003.

[25] W. Rudin, Principles of Mathematical Analysis. New York: McGraw-Hill, 1953.

[26] J. L. Lions, Optimal Control of Systems Governed by Partial Differential Equations. Berlin, Germany: Springer-Verlag, 1971.

[27] K. Hoffman, Analysis in Euclidean Space. Englewood Cliffs, NJ: Prentice-Hall, 1975.

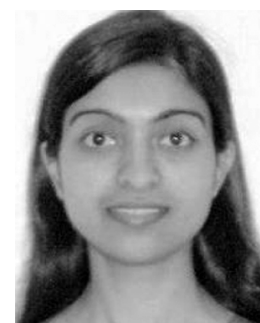

Shrutivandana Sharma received the B.Tech. degree in electrical engineering from the Indian Institute of Technology, Kanpur, India, in 2004.

She is a Ph.D. degree candidate in the Department of Electrical Engineering and Computer Science-Systems, University of Michigan, Ann Arbor. Her research interests are in decentralized decision/resource allocation problems arising in networks and multiagent systems and in the application of ideas from mechanism design and game theory to address these problems.

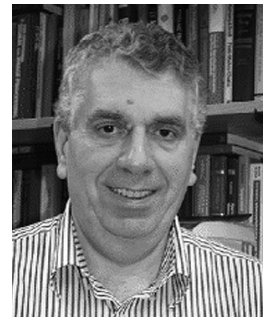

Demosthenis Teneketzis $\left(\mathrm{F}^{\prime} 00\right)$ received the diploma in electrical engineering from the University of Patras, Greece, and the M.S., E.E., and $\mathrm{Ph} . \mathrm{D}$. degrees in electrical engineering from the Massachusetts Institute of Technology, Cambridge.

$\mathrm{He}$ is a professor of Electrical Engineering and Computer Science at the University of Michigan, Ann Arbor. Prior to joining the University of Michigan, he worked for Systems Control Inc., Palo Alto, CA, and Alphatech Inc., Burlington, MA. In Spring and Winter 1992, he was a Visiting Professor with the Swiss Federal Institute of Technology, Zurich, Switzerland. His research interests are in stochastic control, decentralized systems, queuing and communication networks, stochastic scheduling and resource allocation problems, mathematical economics, and discrete event systems. 